\title{
Mesure de l'indice de réfraction par interférométrie en X mous
}

\author{
J. Svatos, F. Polack, D. Joyeux et D. Phalippou
}

IOTA, LURE, CNRS, Bat. 503, 91403 Orsay Cedex, France

\section{ABSTRACT}

We propose a method for measuring directly the refractive index in the soft $x$-ray region using a wavefront division interferometer, the Fresnel twin mirrors. We discuss the design problems and requirements which are imposed by the application of this method to soft X-rays. This method of measurement has the advantage of being insensitive to absorption and is not based on any theory of condensed state. A simulation of the whole experiment is done.

Actuellement, les valeurs de l'indice de réfraction des matériaux dans le domaine $x$ mous $(\lambda \geq 1.5 \mathrm{~nm})$ sont en general obtenues par le calcul, par application des relations de Kramers-Kronig à des mesures d'absorption (1). Ces valeurs sont alors relativement imprécises, en particulier au voisinage de seuils d'absorption.

Nous proposons une mesure directe de l'indice par interférometrie. Nous discutons une réalisation basée sur l'interferomètre à miroirs de Fresnel (Fig.la). On suppose qu'une onde plane monochromatique éclaire un ensemble de deux miroirs, qui sont inclinés l'un par rapport à l'autre de telle façon que les ondes emergentes interfèrent et forment un système de franges. La période des franges ne dépend que du rapport $\lambda / \alpha$, où $\lambda$ est la longueur d'onde et $\alpha$ l'angle entre les deux ondes. Si la phase de l'onde incidente sur un des deux miroirs est décalée par rapport à l'autre, les systèmes de franges présentent un décalage correspondant, (Fig. Ib). Ce changement de phase peut être provoqué par un échantillon placé devant $I^{\prime}$ un des deux miroirs. Il vaut $\Delta \Phi=2 \pi \delta t / \lambda, \delta$ étant la partie réelle de $l^{\prime}$ indice de réfraction $n=1-\delta+i \beta$ du matériau d'épaisseur $t$. L'écart entre les deux systèmes de franges, créés avec et sans échantillon, permet de mesurer $\delta$.

Une simulation numérique de l'expérience a été faite en tenant compte des effets perturbant la mesure: diffraction de Fresnel par des bords des miroirs et de l'échantillon, qualité des miroirs, influence de l'absorption sur le contraste de l'image résultante, forme réelle de l'échantillon.

Les plus grands problèmes proviennent de l'alignement de l'échantillon par rapport aux miroirs. Cette difficulté a été résolue par la fabrication d'un composant optique diffractif spécifique. Un autre problème est la fabrication d'un 
échantilion de forme convenable: deux bords doivent être très bien définis (linearité et largeur de la zone de transition).

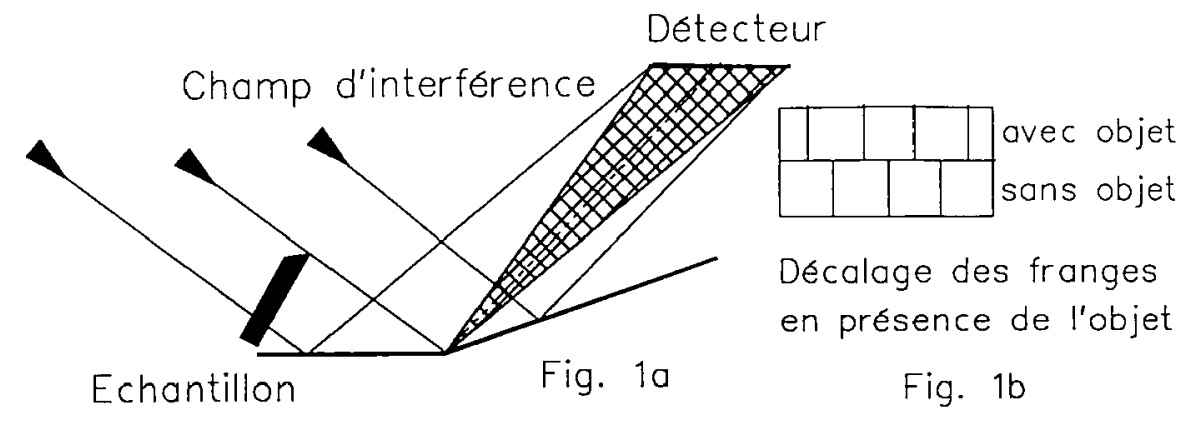

La simulation montre que la diffraction de Fresnel par le bord de I'échantillon et par l'arête commune des miroirs déteriore l'interférogramme résultant. Cependant, ce-dernier présente une qualité suffisante pour appliquer une methode d'analyse globale. On peut placer l'échantillon de telle façon qu'il n'affecte qu' une moitie du champ de franges. Par conséquent, les deux systèmes de franges peuvent être détectés simultanément, par une analyse de type synchrone à l'aide de deux détecteurs. On peut par exemple évaporer une grille absorbante d'un pas correspondant à la période des franges sur un détecteur "temps réel" (diode PIN pour les rayons X mous ou un scintillateur suivi par une photodiode classique). On peut ainsi observer directement la variation des flux détectés en fonction de la position de la grille. Comme les deux interférogrammes sont de forme presque sinusoïdale, on peut determiner leur écart à partir de cette variation de façon précise.

$L^{\prime}$ absorption du rayonnement dans l'échantillon $n^{\prime}$ influence pas directement les résultats de ia mesure, mais elle dimirue le contraste des franges. Comme l'absorption est fonction du produit de l'épaisseur $t$ et de $\beta$, qui lui-même dépend de la longueur d'onde, on doit: soit envisager la fabrication de plusieurs échantillons pour des domaines différents, soit compenser la perte de contraste due à l'absorption dans l'échantilion. Ceci peut être obtenu par exemple par un traitement differencié des miroirs qui améliore le contraste de franges en compensant l'absorption dans l'échantilion par la reflectivité plus faible de l'autre miroix. Il est possible d'obtenir les rapports des reflectivités des miroirs 1:4. Dans ce cas-là, pour la mesure de l'indice du carbone, l'épaisseur varie entre quelques $0,1 \mu \mathrm{m}$ (mesure du côté absorbant du seuil K) et quelques $\mu \mathrm{m}$ (mesure du côté non-absorbant).

$\mathrm{La}$ methode de mesure est directe et elle n'est basée sur aucune theorie (de la dispersion ou autre). De surcroit, l'absorption ne joue aucun rôle direct, donc aucune correction n'est nécessaire. Le dispositif, qui en principe n'est composé que des deux miroirs plans est en cours de fabrication. Les premières expériences sont attendues dans quelques mois.

[1] HENkE et al.,At. Data Nucl. Tables, 27,1-44,1982 